\title{
ON BERNSTEIN-TYPE INEQUALITIES FOR POLYNOMIALS INVOLVING THE POLAR DERIVATIVE
}

\author{
Adil Hussain, Abdullah Mir And Abrar Ahmad
}

\begin{abstract}
In this paper, we establish some upper bound estimates for the polar derivative of a polynomial not vanishing in a disk $|z|<k, k \geqslant 1$ with a zero of multiplicity $s, 0 \leqslant s \leqslant n-1$ at the origin. The obtained results enable us to derive polar derivative analogues of some well known Bernstein-type inequalities as special cases.
\end{abstract}

Mathematics subject classification (2010): 30A10, 30C10, 30D15.

Keywords and phrases: Polar derivative, Bernstein inequality, zeros.

\section{REFERENCES}

[1] A. AzIZ, A refinement of an inequality of S. Bernstein, J. Math. Anal. Appl. 144 (1989), 226-235.

[2] A. AZIZ AND W.M. SHAH, Inequalties for the polar derivative of a polynomial, J. Pure Appl. Math., 29 (1998), 163-173.

[3] S. Bernstein, Sur l'ordre de la meilleure approximation des functions continues par des polynomes de degré donné, Mem. acad. R. Belg., 4 (1912), 1-103.

[4] C. Frappier, Q. I. Rahman and St. Ruscheweyh, New inequalities for polynomials, Trans. Amer. Math. Soc., 288 (1985), 69-99.

[5] N. K. Govil AND Q. I. Rahman, Functions of exponential type not vanishing in a half plane and related polynomials, Trans. Amer. Math. Soc., 137 (1969), 501-517.

[6] P. D. LAX, Proof of a conjecture of P. Erdös on the derivative of a polynomial, Bull. Amer. Math. Soc., 50 (1944), 509-513.

[7] N. A. Rather And M. A. Shah, On the derivative of a polynomial, Appl. Math., 3 (2012), 746749.

[8] S. Hans, D. Tripathi And B. Tyagi, Some inequalities for the derivative of polynomials, J. Mathematics., 2014, pp. 1-5. 